studies are necessary to clarify the value of olfactory identification testing in predicting the onset of $A D$ and its value as an early marker of cognitive decline. In addition, AD patients are more impaired on odor identification and recognition tasks and Parkinson's Disease (PD) patients on odor detection thresholds tasks, what suggests that PD patients are more impaired on low-level perceptual olfactory tasks whereas $A D$ patients are more strongly impaired on higher-order olfactory tasks involving specific cognitive processes. The results suggest smell tests are a cheaper, simpler to apply and a promising weapon for detecting individuals at risk of dementia.

\title{
522 - Role of Physical Environment on Quality of Life among Older Adults with Dementia in Long-Term Care Facilities in Canada and Sweden: A Longitudinal Study
}

Sook Young Lee, Lillian Hung, Habib Chaudhury

Reduction in competence makes older adults with dementia more sensitive to the influence of the physical environment. The aim of the longitudinal study was to examine whether residents with dementia in long-term facilities with variability in physical environmental characteristics in Vancouver $(\mathrm{N}=11)$, Canada and Stockholm $(\mathrm{N}=13)$, Sweden had a difference in their quality of life (QoL). QoL was assessed using Dementia Care Mapping (DCM) tool three times over one year for the reliability of data. DCM is a technique and observational framework devised to systematically investigate QoL from the perspective of the older adults with dementia. The results of the study demonstrated that the residents with dementia living in a homelike and positive stimulating setting showed a higher level of potential positive engagement, and less agitated and withdrawn behaviors compared to those in the large-scale institutional setting. Residents living in a large-scale institutional setting in Canada showed so far as five times more agitated/distressed behaviors and twice more withdrawal compared to the ones living in a small-scale homelike setting in Sweden. The study supports that the large-scale institutional environment was considerably associated with levels of lower quality of life among the residents with dementia.

\section{3 - The design and implementation of Comprehensive Resilience-building psychosocial Intervention (CREST) for people with memory problems/dementia in the community: a pilot study}

Priscilla Doyle, $B A, M A$

Grace O'Sullivan, $B A, M S c$, $P h D$

Niamh Gallagher, $B A, M A$

Siobhán Smyth, RNT, CPN, RPN, BNS (Hons), PG.Dip CHSE, MSc, PhD

Dympna Casey, RGN, BA, MA, PhD

\section{School of Nursing and Midwifery, National University of Ireland Galway; Galway, Ireland}

Background: In Ireland, approximately 65,000 people live with memory problems/dementia (PWMP). Most live in the community, supported by informal caregivers such as relatives. A comprehensive resilience-building psychosocial intervention (CREST) to strengthen intra- and interpersonal resources was piloted by PWMP and caregiver dyads, local GPs, and the community.

Methodology: An advisory forum of PWMP, caregivers, and dementia advocacy representatives provided guidance on the intervention design and materials (e.g., interview guides), to ensure they addressed the needs of PWMP and caregivers. The 15-week CREST intervention comprised three components: cognitive stimulation therapy for PWMP (CST; 7 weeks), physical exercise for PWMP and partners from the community (8 weeks), and dementia education for key supporters of PWMP: caregivers (6 weeks), GPs, and the community (one-off events). Intervention processes (e.g., 